\title{
Axion Emission Can Explain a New Hard X-Ray Excess from Nearby Isolated Neutron Stars
}

\author{
Malte Buschmann $\oplus^{1,2}$ Raymond T. Co ${ }^{3,4}$ Christopher Dessert, ${ }^{1,5,6}$ and Benjamin R. Safdi $\circledast^{1,5,6}$ \\ ${ }^{1}$ Leinweber Center for Theoretical Physics, University of Michigan, Ann Arbor, Michigan 48109, USA \\ ${ }^{2}$ Department of Physics, Princeton University, Princeton, New Jersey 08544, USA \\ ${ }^{3}$ Leinweber Center for Theoretical Physics, University of Michigan, Ann Arbor, Michigan, 48109 USA \\ ${ }^{4}$ William I. Fine Theoretical Physics Institute, School of Physics and Astronomy, University of Minnesota, \\ Minneapolis, Minnesota 55455, USA \\ ${ }^{5}$ Berkeley Center for Theoretical Physics, University of California, Berkeley, California 94720, USA \\ ${ }^{6}$ Theoretical Physics Group, Lawrence Berkeley National Laboratory, Berkeley, California 94720, USA
}

(Received 18 October 2019; revised 4 November 2020; accepted 22 December 2020; published 12 January 2021)

Axions may be produced thermally inside the cores of neutron stars (NSs), escape the stars due to their feeble interactions with matter, and subsequently convert into x rays in the magnetic fields surrounding the stars. We show that a recently discovered excess of hard $\mathrm{x}$-ray emission in the $2-8 \mathrm{keV}$ energy range from the nearby magnificent seven isolated NSs could be explained by this emission mechanism. These NSs are unique in that they had previously been expected to only produce observable flux in the UV and soft x-ray bands from thermal surface emission at temperatures $\sim 100 \mathrm{eV}$. No conventional astrophysical explanation of the magnificent seven hard x-ray excess exists at present. We show that the hard x-ray excess may

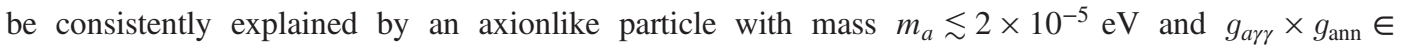
$\left(2 \times 10^{-21}, 10^{-18}\right) \mathrm{GeV}^{-1}$ at $95 \%$ confidence, accounting for both statistical and theoretical uncertainties, where $g_{a \gamma \gamma}\left(g_{\text {ann }}\right)$ is the axion-photon (axion-neutron) coupling constant.

DOI: 10.1103/PhysRevLett.126.021102

Neutron stars (NSs) have long been recognized as excellent laboratories for searching for new light and weakly coupled particles of nature. This is because such particles may be produced abundantly in the hot cores of the NSs, escape, and thus provide a pathway by which the NSs may cool. Some of the strongest constraints on the ultralight pseudo-scalar particles known as axions arise from NS cooling [1-5]. Axions may be produced through nucleon bremsstrahlung in various combinations of proton and neutron scattering in the NS cores [6,7]. It has also been suggested the axions produced in the NS cores may convert into $x$ rays in the magnetospheres surrounding the NSs and that these $\mathrm{x}$ rays may be observable [8-10].

In this work we provide a consistent interpretation of the recently observed hard $\mathrm{x}$-ray excess from the nearby magnificent seven (M7) X-ray dim isolated NSs [11] in the context of an axion model. Reference [11] found significant excesses of hard $\mathrm{x}$-ray emission, in the 2$8 \mathrm{keV}$ energy range, from the M7 using data from the XMM-Newton and Chandra X-ray telescopes. In particular,

Published by the American Physical Society under the terms of the Creative Commons Attribution 4.0 International license. Further distribution of this work must maintain attribution to the author(s) and the published article's title, journal citation, and DOI. Funded by SCOAP ${ }^{3}$.
Ref. [11] found that the NS RX J1856.6 - 3754 (J1856) has around a $5 \sigma$ excess, RX J0420.0-5022 (J0420) has a $\sim 3 \sigma$ excess, and RX J1308.6 + 2127 (J1308) has a $\sim 2 \sigma$ excess. The NSs RX J2143.0+0654 (J2143) and RX J0720.4 - 3125 (J0720) have marginal $\sim 1 \sigma$ excesses, while RX J1605.3 + 3249 (J1605) has a small deficit and is consistent with zero hard X-ray flux. We show that the M7 hard x-ray data may be explained in the context of an axion model where the axion couples to both nucleons and photons. The fact that hard $\mathrm{x}$-ray emission is observed from some NSs and not others is consistent with the axion model because (i) the exposure times vary across the M7, (ii) the predicted fluxes at fixed axion parameters vary between NSs, given their different properties, and (iii) these properties are uncertain at present. We also provide one of the most competitive constraints to date on the axion-photon times axion-nucleon coupling for axion masses $m_{a} \lesssim 10^{-4} \mathrm{eV}$.

The M7 were discovered in soft x rays with the ROSAT All Sky Survey (see, e.g., Ref. [12]). Their soft spectra are well described by near-thermal distributions with surface temperatures $\sim 50-100 \mathrm{eV}$. No nonthermal emission, for example, in radio, has been previously observed from the NSs. As such, they are expected to produce negligible hard $\mathrm{x}$-ray flux, making them background-free from the point of view of the analysis described in this work. Moreover, they are all observed to have strong magnetic fields [13-20] and to be relatively nearby, at distances of order hundreds of pc. 
The quantum chromodynamics (QCD) axion is a hypothetical ultralight particle that solves the strong $C P$ problem of the neutron electric dipole moment [21-24] and may also make up the observed dark matter [25-27]. The QCD axion and axionlike particles (ALPs) more generally also appear to be a relatively generic expectation from string compactifications [28,29], and the ALP masses in particular may be significantly lighter than the $\sim 10^{-4} \mathrm{eV}$ threshold relevant for this work (see, e.g., Refs. [30,31]). Both the QCD axion and ALPs are expected to couple derivatively to matter and also couple to electromagnetism, allowing them to be produced inside of the hot NSs and converted into photons in the strong magnetic fields surrounding the NSs. Thus in this work we refer to both particles simply as axions. Intriguingly, recent string theory constructions suggest that the ALP photon couplings may be slightly smaller than current limits and within reach of the search discussed in this work [32].

Axions have also been discussed in the context of white dwarf, red giant, and horizontal-branch (HB) star cooling [33-42]. In white dwarf (WD) and red giant stars the dominant production modes involve the axion-electron coupling while in HB stars the axion-photon production dominates. Recently it was proposed that $\mathrm{x}$-ray observations of magnetic WD stars may probe axion scenarios, since the hot axions produced in the WD cores may convert into $x$ rays in the magnetic fields surrounding the WDs [43]. Axion-photon conversion within NS magnetospheres has been discussed recently in the context of dark matter axions [44-47]. Axions and axion dark matter are also the subject of considerable laboratory searches [48-63].

This work takes the M7 hard x-ray spectra from Ref. [11] as a starting point. Additional analysis details and systematic tests relevant for the axion model are presented in the Supplemental Material [64].

Axion-induced $x$-ray flux from NSs.-The central idea behind the proposed signal is that while the cores of the M7 are quite hot $(T \sim 1-10 \mathrm{keV})$ the surfaces are relatively cool with $T \sim 0.1 \mathrm{keV}$. Axions may be emitted from hot NS interiors, escape the NSs, and then convert into hard $\mathrm{x}$ rays in the strong magnetic fields surrounding the NSs. To calculate the expected signal we both account for the axion production rate in the NS cores and the conversion probability in the magnetospheres.

The axions are produced in the NS cores through the axion couplings to fermionic matter. The coupling of the axion $a$ to a fermion $\psi_{f}$ is denoted by (see, e.g., Ref. [87]) $\mathcal{L}=\left(C_{f} / 2 f_{a}\right) \bar{\psi}_{f} \gamma^{\mu} \gamma_{5} \psi_{f} \partial_{\mu} a$, with $f_{a}$ the axion decay constant. Scattering amplitudes involving this operator are generally functions of the dimensionless coupling combination $g_{\text {aff }}=C_{f} m_{f} / f_{a}$, with $m_{f}$ the fermion mass and $C_{f}$ the dimensionless Lagrangian coupling (we use $C_{p}$ and $C_{n}$ for the proton and neutron, respectively). Note that the axion-fermion operators are generated in the infrared through the renormalization group, given an axion-photon coupling, even if such operators are absent in the ultraviolet [43,88,89].

The axion production mechanisms relevant for this work mostly occur in the NS core through axion bremsstrahlung in fully degenerate nucleon-nucleon scattering $N_{1} N_{2} \rightarrow$ $N_{1} N_{2} a$, where the $N_{1,2}$ are either neutrons or protons. The emissivities for these processes are functions of the couplings $g_{\text {ann }}, g_{\text {app }}$, the local NS core temperature $T$, and the neutron and proton Fermi momenta (see the Supplemental Material [64] and Refs. [6,7]). As shown in Ref. [6], the local energy spectrum of axions emitted from these processes follows the modified thermal distribution $d F / d E \propto$ $z^{3}\left(z^{2}+4 \pi^{2}\right) /\left(e^{z}-1\right)$, where $z=E / T, E$ is the local axion energy, and $F$ is flux. We note that the nucleon bremsstrahlung rates may be suppressed at low temperatures, below the critical temperature for Cooper pair formation, by nucleon superfluidity [2,90]. Recent analyses of NS cooling [91] indicate that the critical temperatures are likely too low to be relevant for this work, and so we ignore possible nucleon superfluidity in our fiducial analyses. However, given that the critical temperatures are uncertain at present, we discuss their possible effects in depth in the Supplemental Material [64].

To compute the production rates in the NS cores, given the emissivity formulas, we need to know the temperature profiles in the cores, the metric, the critical temperature profiles (if including superfluidity), and the profiles of neutron and proton Fermi momenta, which all depend on the NS equation of state (EOS). We use the code package NSCOOl [92] to perform the thermal evolution of the NSs, in full general relativity and assuming spherical symmetry. For our fiducial analysis we use the APR EOS [93] and assume NS masses of $1.4 M_{\odot}$. The thermal evolution is used to obtain a relation between the effective surface temperature and the isothermal core temperature $T_{b}^{\infty}$, which is the redshifted temperature infinitely far from the NS's potential well. The surface temperatures and associated statistical uncertainties are taken from the recent compilation in Ref. [91], which accounts for the effects of NS atmospheres in lowering the surface temperature for many NSs relative to the observed single-blackbody temperature.

The relation between the surface and core temperatures is known to be strongly affected by accretion and magnetic fields, and moreover strong magnetic fields may make the surface temperature inhomogeneous (see, e.g., Ref. [94]). In fact it is the anisotropic surface temperatures that are thought to lead to the observed x-ray pulsations of the M7 [95]. Additionally, NS atmospheres may distort the spectra away from perfect blackbodies [96,97]. We account for these possibilities through a systematic uncertainty on the core temperatures, as described in the Supplemental Material [64]. We combine all $T_{b}^{\infty}$ uncertainties, statistical and systematic, into single Gaussian priors, with standard deviations given in Table I, with the restriction that $T_{b}^{\infty}>0$.

The core temperatures may also be estimated from the kinematic ages of the NSs. The local temperature at the 
TABLE I. The M7 properties used in this work. The magnetic field strength at the pole $B_{0}$ is in $10^{13} \mathrm{G}$; the surface temperature at infinity $T_{s}^{\infty}$ is in $\mathrm{eV}$; the core boundary temperature at infinity $T_{b}^{\infty}$ is in keV; the distance $d$ is in pc; the hard X-ray intensity $I_{x-8}$ is in $10^{-15} \mathrm{erg} / \mathrm{cm}^{2} / \mathrm{s}$, integrated from $x \mathrm{keV}$ to $8 \mathrm{keV}$, with $x=4$ for all NSs but J0420 and J1856, for which $x=2$. We obtain the NS properties from the catalog of cooling NSs [91], and the $I_{x-8}$ 's from Ref. [11].

\begin{tabular}{lrrrrrc}
\hline \hline Name & $B_{0}$ & $T_{s}^{\infty}$ & $T_{b}^{\infty}$ & $d$ & $I_{x-8}$ & Refs. \\
\hline J0806 & 5.1 & $100 \pm 10$ & $15 \pm 9$ & $240 \pm 25$ & $0.0_{-0.3}^{+1.6}$ & {$[18,98,99]$} \\
J1856 & 2.9 & $50 \pm 14$ & $5 \pm 3$ & $123 \pm 13$ & $1.5_{-0.6}^{+0.7}$ & {$[16,100-103]$} \\
J0420 & 2.0 & $45 \pm 10$ & $3 \pm 2$ & $345 \pm 200$ & $0.7_{-0.5}^{+1.0}$ & {$[19,98,99]$} \\
J1308 & 6.8 & $70 \pm 20$ & $8 \pm 6$ & $380 \pm 30$ & $2.3_{-1.7}^{+1.8}$ & {$[15,104,105]$} \\
J0720 & 6.8 & $92 \pm 10$ & $13 \pm 8$ & $286 \pm 25$ & $0.9_{-1.6}^{+1.1}$ & {$[14,106]$} \\
J1605 & 2.0 & $78 \pm 42$ & $9 \pm 11$ & $174 \pm 52$ & $-0.5_{-0.7}^{+1.3}$ & {$[20,107]$} \\
J2143 & 4.0 & $72 \pm 32$ & $8 \pm 8$ & $430 \pm 200$ & $3.1_{-3.4}^{+3.0}$ & {$[17,99,108]$} \\
\hline \hline
\end{tabular}

outer boundary of the core $T_{b}$ is expected to evolve as $T_{b} \approx 10^{9}(t / \mathrm{yr})^{-1 / 6} \mathrm{~K}$ over times $t \gg \mathrm{yr}$, neglecting effects such as ambipolar diffusion, which may provide additional heating to the core [109]. The kinematic core temperature estimates agree with those in Table I within uncertainties when the NS ages are available, though there are minor differences which, as shown in the Supplemental Material, Fig. S8 [64], lead to slightly lower inferred axion couplings when using core-temperature priors from age estimates.

We then consider the conversion of the axions into $\mathrm{x}$ rays in the NS magnetic fields. Here we follow closely the framework outlined in Ref. [43] for axion-photon conversion in WD magnetospheres. The axion-photon mixing is induced through the operator $\mathcal{L}=-g_{\text {ary }} a F \tilde{F} / 4$, where $F$ is the electromagnetic field strength tensor, $\tilde{F}$ is its dual field, and $g_{a \gamma \gamma}$ is the axion-photon mixing parameter. The parameter $g_{a \gamma \gamma}$ is related to $f_{a}$ through the relation $g_{a \gamma \gamma}=C_{\gamma} \alpha_{\mathrm{EM}} /\left(2 \pi f_{a}\right)$, with $\alpha_{\mathrm{EM}}$ the fine structure constant and $C_{\gamma}$ a dimensionless coupling constant. In the presence of a strong magnetic field this operator may cause an initially pure axion state to rotate into an electromagnetic wave polarized parallel to the external magnetic field. However, the axion-photon conversion is suppressed by the Euler-Heisenberg term for strong field quantum electrodynamics [9].

In the limit of low axion mass, which for our applications is roughly $m_{a} \lesssim\left(\omega R_{\mathrm{NS}}^{-1}\right)^{1 / 2}$ (and approximately $10^{-4} \mathrm{eV}$ at axion frequencies $\omega \sim \mathrm{keV}$ and NS radii $R_{\mathrm{NS}} \sim 10 \mathrm{~km}$ ), the conversion probability $p_{a \rightarrow \gamma}$ is approximately $[9,10,43]$

$$
\begin{aligned}
p_{a \rightarrow \gamma} \approx & 1.5 \times 10^{-4}\left(\frac{g_{a \gamma \gamma}}{10^{-11} \mathrm{GeV}^{-1}}\right)^{2}\left(\frac{1 \mathrm{keV}}{\omega}\right)^{4 / 5} \\
& \times\left(\frac{B_{0}}{10^{13} \mathrm{G}}\right)^{2 / 5}\left(\frac{R_{\mathrm{NS}}}{10 \mathrm{~km}}\right)^{6 / 5} \sin ^{2 / 5} \theta,
\end{aligned}
$$

independent of the axion mass. Above, $B_{0}$ is the surface magnetic field strength at the magnetic pole, assuming a dipole field configuration, and $\theta$ is the polar angle from the magnetic axis. At large axion masses the conversion probability becomes additionally suppressed and must be computed numerically (see, e.g., Ref. [43]).

We assume dipolar magnetic field strengths calculated from the spin-down of the NSs [13-19] via magneto-dipole radiation. (Note that the statistical uncertainties on the dipole field strengths are subleading.) In the case of J1605, there is no spin-down measurement and we adopt $2 \times$ $10^{13} \mathrm{G}$ as considered in Ref. [20]. Measurements of the magnetic field from spectral fitting of cyclotron resonance lines or atmosphere models generally predict larger fields, which we consider in the Supplemental Material [64]. We account for the unknown alignment angle $\theta$ by profiling over $\theta$ with a flat prior.

Data analysis.-Ref. [11] analyzed all available archival data from XMM-Newton and Chandra towards each of the M7 for evidence of hard X-ray emission. For XMM-Newton they reprocessed data from both the MOS and PN cameras and treated these datasets independently, since they are subject to different sources of uncertainty from, e.g., pileup. The data were binned into three high-energy bins from 2-4, 4-6, and 6-8 keV. Reference [11] computed likelihood profiles for flux from the M7 in each one of these energy bins; these likelihoods are provided as supplemental data in Ref. [11] and are the starting points for the analyses presented in this work. As an illustration, in Fig. 1 we show the energy spectrum from J1856, which is the NS with the most significant hard x-ray excess. Note that we show the best-fit fluxes and associated $68 \%$ confidence intervals from the joint analyses over all three cameras.

Reference [11] showed that the $2-4 \mathrm{keV}$ energy bin may be contaminated by the high-energy tail of the thermal emission from the NS surfaces, depending on the atmosphere model, for all NSs except J1856 and J0420. The predicted thermal surface emission is negligible for all NSs

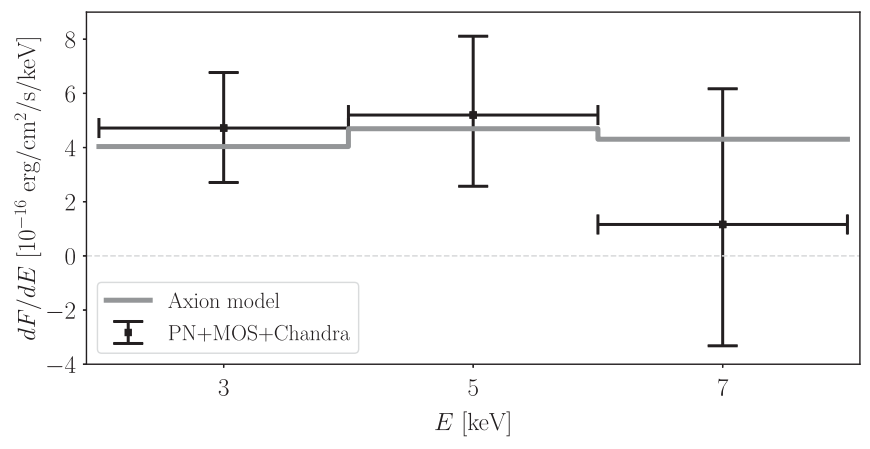

FIG. 1. The energy spectrum from 2 to $8 \mathrm{keV}$ for NS J1856 as measured by combining PN, MOS, and Chandra data, with $68 \%$ statistical uncertainties [11]. We also show the best-fit axion model spectrum from a fit to this NS only, with the core temperature fixed to the central value in Table I. 


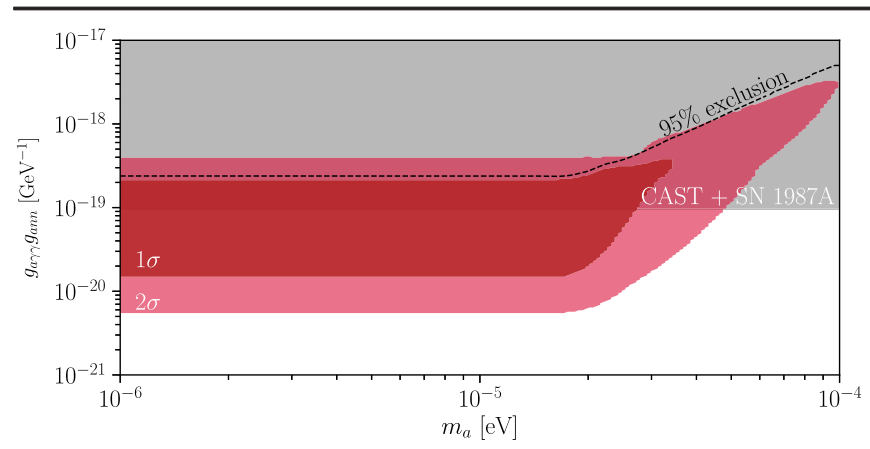

FIG. 2. $95 \%$ exclusion limit and best fit 1 and $2 \sigma$ regions from a joint likelihood analysis over all of the M7 and combining PN, MOS, and Chandra data. We compare our result to existing limits from CAST2017 + NS cooling. All curves and regions continue to arbitrarily small $m_{a}$. Note that the QCD axion model is too weakly coupled to appear in this figure. Accounting for systematic uncertainties may allow for smaller values of $g_{a \gamma \gamma} g_{\text {ann }}$, by approximately an order of magnitude, as discussed in the Supplemental Material [64].

in the last two energy bins. As such in this analysis we use all three available energy bins for J1856, which has by far the most exposure time of all M7, and J0420, but only the last two energy bins for the other five NSs. Reference [11] only provides Chandra data for J1856, J0420, and J0806, because for the other NSs they found that pileup may affect the observed high-energy spectrum. For J2143 only PN data is available.

We interpret the M7 hard x-ray spectra in the context of the axion model by using a joint likelihood over all of the M7 and available datasets with a frequentist profile likelihood analysis procedure. Our parameters of interest are $\left\{m_{a}, g_{\text {ary }}, g_{\text {ann }}, g_{\text {app }}\right\}$ and our nuisance parameters, which describe uncertain aspects of the NSs, are the set of parameters $\left\{\theta, d, T_{b}^{\infty}\right\}$ for each NS, where $d$ is distance. Each of the nuisance parameters is taken to have a Gaussian prior with uncertainty given in Table I, except for $\theta$, which is given a flat prior from 0 to $\pi$. Uncertainties arising from the NS superfluidity model are described in the Supplemental Material [64]. For our fiducial analysis we fix $g_{\text {app }}=g_{\text {ann }}$. We construct a joint likelihood over all of the M7 and available datasets, and we use this likelihood to constrain our parameters of interest.

Results.-The resulting best-fit parameter space in the $m_{a}-g_{a \gamma \gamma} g_{\text {ann }}$ plane and $95 \%$ one-sided upper limit are shown in Fig. 2. Interpreting the data in the context of the axion model, we find approximately $5 \sigma$ evidence for the axion-induced flux over the null hypothesis of no nonthermal hard x-ray flux from the M7. The global fit prefers a low axion mass and a coupling at and slightly below previous limits, which are also indicated. In particular we combine the CAST constraints on $g_{a \gamma \gamma}$ $\left(g_{\text {ary }}<6.6 \times 10^{-11} \mathrm{GeV}^{-1}\right.$ at low masses) [110] with the SN 1987A constraints on $g_{\text {ann }}$, taking $g_{\text {app }}=g_{\text {ann }}, g_{\text {ann }}<$ $1.4 \times 10^{-9}$ [111] (but see Ref. [112] which question these

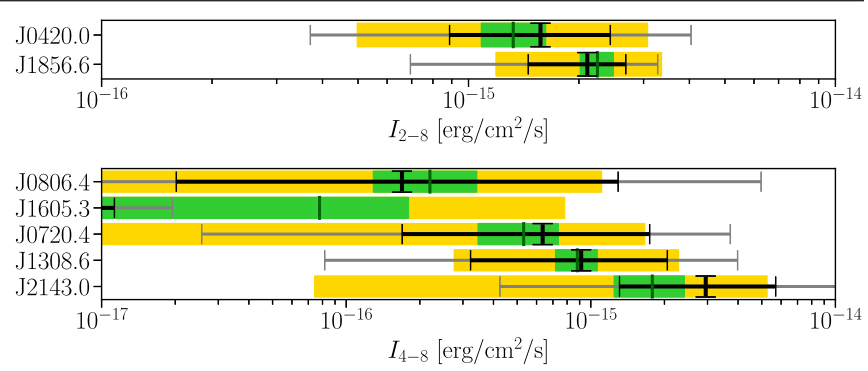

FIG. 3. Best-fit intensities $I_{2-8}$ and $I_{4-8}$ for all M7. The green (yellow) bands indicate the $68 \%$ (95\%) confidence intervals from the x-ray intensity measurements, with best-fit intensities marked by vertical green lines. Black and gray error bands denote the $68 \%$ and $95 \%$ confidence intervals for the axion model predictions at the global best-fit coupling $g_{a \gamma \gamma} g_{\text {ann }}$ and $m_{a} \ll 10^{-5} \mathrm{eV}$, with uncertainties arising from uncertain aspects of the NSs.

constraints). Constraints on $g_{\text {ann }}$ from cooling of the NS Cas A $[2,4,5]$ may all be relevant, though these constraints are subject to both instrumental [113] and theoretical systematic uncertainties. Thus the current constraints on $g_{a \gamma \gamma} g_{\text {ann }}$ in Fig. 2 should be taken as suggestive.

It is interesting to investigate whether the high-energy flux observed between the individual NSs is consistent with the expectation from the axion hypothesis. In Fig. 3 we show the observed intensities $I_{2-8}\left(I_{4-8}\right)$ between $2-8 \mathrm{keV}$ (4-8 keV) for each of the M7 after combining the MOS, $\mathrm{PN}$, and Chandra datasets. These intensities are determined by fitting the low-mass axion spectral model uniquely to the data from each NS, with model parameters $T_{b}^{\infty}$ and $I_{2-8}$ $\left(I_{4-8}\right)$. Note that for the NSs where we include the $2-4 \mathrm{keV}$ energy bin we report $I_{2-8}$, while for those where we do not include this bin we instead report $I_{4-8}$. (We obtain qualitatively similar results if we only use the $4-8 \mathrm{keV}$ bins for all NSs, as shown in the Supplemental Material [64].) The green (yellow) bands indicate the 68\% (95\%) confidence intervals for the intensities from the x-ray measurements. The black and gray error bands, on the other hand, denote the $68 \%$ and $95 \%$ confidence intervals for the axion model predictions, fixing the axion model parameters at the bestfit point from the global fit, $g_{a \gamma \gamma} g_{\text {ann }} \approx 4 \times 10^{-20} \mathrm{GeV}^{-1}$ with $m_{a} \ll 10^{-5} \mathrm{eV}$, and profiling over the nuisance parameters. The uncertainties in the model prediction arise primarily from the nuisance parameters describing the unknown properties of the M7, as described above, while the uncertainties on the measured intensity values are purely statistical in nature.

The observed intensities are consistent with expectations from the axion model. Additionally, there are sources of uncertainty on the axion model predictions for the individual NSs beyond those shown in Fig. 3, arising from for example nucleon superfluidity, the EOS, and the inference of the core temperatures. For example, as we show in the Supplemental Material [64] with alternate core-temperature 
J0420.0-

J1856.6-

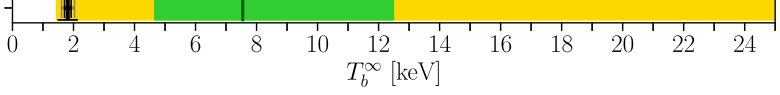

FIG. 4. As in Fig. 3 but for the best-fit core temperature $T_{b}^{\infty}$ for $\mathrm{J} 1856$ and $\mathrm{J} 0420$.

models, based on ages rather than surface temperatures, the best-fit couplings could be as low as $g_{a \gamma \gamma} g_{\text {ann }} \approx$ $2 \times 10^{-21} \mathrm{GeV}^{-1}$.

We also investigate whether the observed spectra from the two high-significance detections in J1856 and J0420 are consistent with the axion model expectation. In Fig. 4 we show the best-fit core temperatures $T_{b}^{\infty}$ measured from fitting the axion model, with $m_{a} \ll 10^{-5} \mathrm{eV}$, to the x-ray data between 2 and $8 \mathrm{keV}$. We note that the NS with the best-determined spectral shape is J1856, which has the most significant detection. In Fig. 1 we show the best-fit model prediction for this NS compared to the observed spectrum. The axion model appears to reproduce the spectral shape found in the data.

Discussion.-In this work we presented results of a search for hard x-ray emission arising from axions in the M7 NSs. We showed that the M7 hard x-ray excess observed in Ref. [11] may be interpreted in the context of the axion model.

Alternative explanations for the hard $\mathrm{x}$-ray emission exist, but they are not compelling [11]. For example, some of the observations may be affected by pileup due to the high flux of soft, thermal $x$ rays, though these effects seem insufficient to explain the observed hard x-ray flux [11]. For the XMM-Newton data in particular, unresolved astrophysical point sources near the source of interest could also bias the observed spectrum, though the fact that consistent spectra are observed with Chandra, which has over an order of magnitude better angular resolution, provides evidence that this is at least not the sole explanation for the excess. Hard nonthermal x-ray emission is observed generically from pulsars, and one possibility is that the observed hard $\mathrm{x}$-ray flux from the M7 arises from the traditional nonthermal emission mechanisms (e.g., synchrotron emission) that are present in other pulsars. On the other hand, this emission is often accompanied by nonthermal radio emission, which is not observed for the M7 [114], and also the spin-down luminosity seems insufficient for most of the M7 for this to be an appreciable source of flux [11]. Accretion of the interstellar medium may also be a source of $\mathrm{x}$ rays from the $\mathrm{M} 7$, though this is typically thought to produce flux at much softer energies if at all (see, e.g., Ref. [115]).

Observations at higher energies by, e.g., NuSTAR of J1856 and J0420 in particular may help discriminate the axion explanation of the excess from other explanations. This is because the predicted axion spectrum in the energy range from $\sim 10-60 \mathrm{keV}$ is unique and potentially includes a significant enhancement due to Cooper pair-breakingformation processes, depending on the superfluidity model. (See the Supplemental Material [64] for details, where we also show that the Cooper pair processes could enhance the flux below $10 \mathrm{keV}$ as well.) The axion-induced flux should also pulsate at the NS period, and this may be measurable with future instruments such as Athena that can acquire better statistics. Athena will have similar angular resolution to Chandra while also being significantly less affected by pileup [116]. X-ray observations of magnetic white dwarf stars [43], the magnetized intracluster medium [117], or nearby bright galaxies [118] could also help constrain or provide additional evidence for the best-fit axion from this work. The best-fit axion parameter space from this work may also be probed with next-generation light-shiningthrough-walls experiments like ALPS II [119] and helioscopes like IAXO [120]. In summary, if the M7 hard x-ray excess is due to axions, then a variety of near-term measurements should be able to conclusively establish a discovery.

We are grateful to Y. Kahn for collaboration in the early stages of this work and comments on the manuscript and to J. Foster, M. Reynolds, O. Gnedin, H. Günther, D. Hooper, A. Long, A. Ringwald, and D. Yakovlev for useful discussions and comments. We thank the anonymous referees for useful suggestions. This work was supported in part by the DOE Early Career Grant No. DESC0019225 and the DOE grant DE-SC0011842 (R.C.) at the University of Minnesota and through computational resources and services provided by Advanced Research Computing at the University of Michigan, Ann Arbor. C. D. was partially supported by the Leinweber Graduate Fellowship at the University of Michigan, Ann Arbor. This work was performed in part at the Aspen Center for Physics, which is supported by the National Science Foundation Grant No. PHY-1607611, and in part at the Mainz Institute for Theoretical Physics (MITP) of the Cluster of Excellence PRISMA+ (Project ID 39083149). We also acknowledge the Munich Institute for Astro- and Particle Physics (MIAPP) of the DFG Excellence Cluster Origins along with the CERN Theory department for hospitality during the completion of this work.

[1] H. Umeda, N. Iwamoto, S. Tsuruta, L. Qin, and K. Nomoto, in Workshop on Neutron Stars and Pulsars: Thirty Years after the Discovery Tokyo, Japan (World Scientific, 1998).

[2] J. Keller and A. Sedrakian, Nucl. Phys. A897, 62 (2013).

[3] L. B. Leinson, J. Cosmol. Astropart. Phys. 08 (2014) 031.

[4] A. Sedrakian, Phys. Rev. D 93, 065044 (2016).

[5] A. Sedrakian, Phys. Rev. D 99, 043011 (2019).

[6] N. Iwamoto, Phys. Rev. Lett. 53, 1198 (1984).

[7] R. P. Brinkmann and M. S. Turner, Phys. Rev. D 38, 2338 (1988). 
[8] D. E. Morris, Phys. Rev. D 34, 843 (1986).

[9] G. Raffelt and L. Stodolsky, Phys. Rev. D 37, 1237 (1988).

[10] J.-F. Fortin and K. Sinha, J. High Energy Phys. 06 (2018) 048.

[11] C. Dessert, J. W. Foster, and B. R. Safdi, Astrophys. J. 904, 42 (2020).

[12] F. Haberl, Astrophys. Space Sci. 308, 181 (2007).

[13] D. L. Kaplan and M. H. van Kerkwijk, Astrophys. J. 628, L45 (2005).

[14] V. Hambaryan, V. Suleimanov, F. Haberl, A. D. Schwope, R. Neuhäuser, M. Hohle, and K. Werner, Astron. Astrophys. 601, A108 (2017).

[15] D. L. Kaplan and M. H. van Kerkwijk, Astrophys. J. 635, L65 (2005).

[16] M. H. van Kerkwijk and D. L. Kaplan, Astrophys. J. 673, L163 (2008)

[17] D. L. Kaplan and M. H. van Kerkwijk, Astrophys. J. 692, L62 (2009).

[18] D. L. Kaplan and M. H. van Kerkwijk, Astrophys. J. 705, 798 (2009).

[19] D. L. Kaplan and M. H. van Kerkwijk, Astrophys. J. 740, L30 (2011).

[20] C. Malacaria et al., Astrophys. J. 880, 74 (2019).

[21] R. D. Peccei and H. R. Quinn, Phys. Rev. D 16, 1791 (1977).

[22] R. D. Peccei and H. R. Quinn, Phys. Rev. Lett. 38, 1440 (1977).

[23] S. Weinberg, Phys. Rev. Lett. 40, 223 (1978).

[24] F. Wilczek, Phys. Rev. Lett. 40, 279 (1978).

[25] J. Preskill, M. B. Wise, and F. Wilczek, Phys. Lett. 120B, 127 (1983).

[26] L. F. Abbott and P. Sikivie, Phys. Lett. 120B, 133 (1983).

[27] M. Dine and W. Fischler, Phys. Lett. 120B, 137 (1983).

[28] P. Svrcek and E. Witten, J. High Energy Phys. 06 (2006) 051.

[29] A. Arvanitaki, S. Dimopoulos, S. Dubovsky, N. Kaloper, and J. March-Russell, Phys. Rev. D 81, 123530 (2010).

[30] L. Hui, J. P. Ostriker, S. Tremaine, and E. Witten, Phys. Rev. D 95, 043541 (2017).

[31] M. J. Stott and D. J. E. Marsh, Phys. Rev. D 98, 083006 (2018).

[32] J. Halverson, C. Long, B. Nelson, and G. Salinas, Phys. Rev. D 100, 106010 (2019).

[33] G. G. Raffelt, Phys. Lett. 166B, 402 (1986).

[34] J. Isern, E. Garcia-Berro, S. Torres, and S. Catalan, Astrophys. J. 682, L109 (2008).

[35] J. Isern, S. Catalan, E. Garcia-Berro, and S. Torres, J. Phys. Conf. Ser. 172, 012005 (2009).

[36] J. Isern, E. Garcia-Berro, L. G. Althaus, and A. H. Corsico, Astron. Astrophys. 512, A86 (2010).

[37] M. M. M. Bertolami, B. E. Melendez, L. G. Althaus, and J. Isern, J. Cosmol. Astropart. Phys. 10 (2014) 069.

[38] A. Ayala, I. Domínguez, M. Giannotti, A. Mirizzi, and O. Straniero, Phys. Rev. Lett. 113, 191302 (2014).

[39] J. Redondo, J. Cosmol. Astropart. Phys. 12 (2013) 008.

[40] N. Viaux, M. Catelan, P. B. Stetson, G. G. Raffelt, J. Redondo, A. A. R. Valcarce, and A. Weiss, Phys. Rev. Lett. 111, 231301 (2013).

[41] M. Giannotti, I. Irastorza, J. Redondo, and A. Ringwald, J. Cosmol. Astropart. Phys. 05 (2016) 057.
[42] M. Giannotti, I. G. Irastorza, J. Redondo, A. Ringwald, and K. Saikawa, J. Cosmol. Astropart. Phys. 10 (2017) 010.

[43] C. Dessert, A. J. Long, and B. R. Safdi, Phys. Rev. Lett. 123, 061104 (2019).

[44] M. S. Pshirkov and S. B. Popov, J. Exp. Theor. Phys. 108, 384 (2009).

[45] A. Hook, Y. Kahn, B. R. Safdi, and Z. Sun, Phys. Rev. Lett. 121, 241102 (2018).

[46] B. R. Safdi, Z. Sun, and A. Y. Chen, Phys. Rev. D 99, 123021 (2019).

[47] T. D. P. Edwards, M. Chianese, B. J. Kavanagh, S. M. Nissanke, and C. Weniger, Phys. Rev. Lett. 124, 161101 (2020).

[48] T. M. Shokair et al., Int. J. Mod. Phys. A 29, 1443004 (2014).

[49] N. Du, N. Force, R. Khatiwada, E. Lentz, R. Ottens et al. (ADMX Collaboration), Phys. Rev. Lett. 120, 151301 (2018).

[50] B. M. Brubaker, L. Zhong, Y. V. Gurevich, S. B. Cahn, S. K. Lamoreaux et al., Phys. Rev. Lett. 118, 061302 (2017).

[51] S. Al Kenany et al., Nucl. Instrum. Methods Phys. Res., Sect. A 854, 11 (2017).

[52] B. M. Brubaker, L. Zhong, S. K. Lamoreaux, K. W. Lehnert, and K. A. van Bibber, Phys. Rev. D 96, 123008 (2017).

[53] A. Caldwell, G. Dvali, B. Majorovits, A. Millar, G. Raffelt, J. Redondo, O. Reimann, F. Simon, and F. Steffen (MADMAX Working Group), Phys. Rev. Lett. 118, 091801 (2017).

[54] Y. Kahn, B. R. Safdi, and J. Thaler, Phys. Rev. Lett. 117, 141801 (2016).

[55] J. W. Foster, N. L. Rodd, and B. R. Safdi, Phys. Rev. D 97, 123006 (2018).

[56] J. L. Ouellet et al., Phys. Rev. Lett. 122, 121802 (2019).

[57] S. Chaudhuri, P. W. Graham, K. Irwin, J. Mardon, S. Rajendran, and Y. Zhao, Phys. Rev. D 92, 075012 (2015).

[58] M. Silva-Feaver et al., IEEE Trans. Appl. Supercond. 27, 1400204 (2016).

[59] D. Budker, P. W. Graham, M. Ledbetter, S. Rajendran, and A. O. Sushkov, Phys. Rev. X 4, 021030 (2014).

[60] R. Bähre et al., J. Instrum. 8, T09001 (2013).

[61] Z. Bogorad, A. Hook, Y. Kahn, and Y. Soreq, Phys. Rev. Lett. 123, 021801 (2019).

[62] R. Janish, V. Narayan, S. Rajendran, and P. Riggins, Phys. Rev. D 100, 015036 (2019).

[63] M. Lawson, A. J. Millar, M. Pancaldi, E. Vitagliano, and F. Wilczek, Phys. Rev. Lett. 123, 141802 (2019).

[64] See Supplemental Material at http://link.aps.org/ supplemental/10.1103/PhysRevLett.126.021102, which includes Refs. [65-86], for our determination of the core temperature uncertainties, review of the axion production rates, statistical analysis framework, and multiple systematic tests of the fiducial analyses.

[65] E. H. Gudmundsson, C. J. Pethick, and R. I. Epstein, Astrophys. J. 272, 286 (1983).

[66] A. Y. Potekhin, G. Chabrier, and D. G. Yakovlev, Astron. Astrophys. 323, 415 (1997).

[67] M. Bejger and P. Haensel, Astron. Astrophys. 420, 987 (2004). 
[68] N. Iwamoto, Phys. Rev. D 64, 043002 (2001).

[69] D. Page, M. Prakash, J. M. Lattimer, and A. W. Steiner, Phys. Rev. Lett. 106, 081101 (2011).

[70] G. Cowan, K. Cranmer, E. Gross, and O. Vitells, Eur. Phys. J. C 71, 1554 (2011); 73, 2501(E) (2013).

[71] M. Hoffberg, A. E. Glassgold, R. W. Richardson, and M. Ruderman, Phys. Rev. Lett. 24, 775 (1970).

[72] L. Amundsen and E. Ostgaard, Nucl. Phys. A442, 163 (1985).

[73] T. Takatsuka, Prog. Theor. Phys. 48, 1517 (1972).

[74] M. Baldo, J. Cugnon, A. Lejeune, and U. Lombardo, Nucl. Phys. A536, 349 (1992).

[75] O. Elgaroy, L. Engvik, M. Hjorth-Jensen, and E. Osnes, Phys. Rev. Lett. 77, 1428 (1996).

[76] A. Schwenk, B. Friman, and G. E. Brown, Nucl. Phys. A713, 191 (2003).

[77] D. Page, J. M. Lattimer, M. Prakash, and A. W. Steiner, Astrophys. J. Suppl. Ser. 155, 623 (2004).

[78] M. Baldo, J. Cugnon, A. Lejeune, and U. Lombardo, Nucl. Phys. A536, 349 (1992).

[79] K. Oh et al., Astrophys. J. Suppl. Ser. 235, 4 (2018).

[80] R. Krivonos, S. Tsygankov, I. Mereminskiy, A. Lutovinov, S. Sazonov, and R. Sunyaev, Mon. Not. R. Astron. Soc. 470, 512 (2017).

[81] A. Schwenk and B. Friman, Phys. Rev. Lett. 92, 082501 (2004).

[82] R. P. Mignani, D. Vande Putte, M. Cropper, R. Turolla, S. Zane, L. J. Pellizza, L. A. Bignone, N. Sartore, and A. Treves, Mon. Not. R. Astron. Soc. 429, 3517 (2013).

[83] A. D. Schwope, V. Hambaryan, F. Haberl, and C. Motch, Astrophys. Space Sci. 308, 619 (2007).

[84] M. H. van Kerkwijk, D. L. Kaplan, M. Durant, S. R. Kulkarni, and F. Paerels, Astrophys. J. 608, 432 (2004).

[85] N. Tetzlaff, J. G. Schmidt, M. M. Hohle, and R. Neuhäuser, Pub. Astron. Soc. Aust. 29, 98 (2012).

[86] J. Braithwaite, Mon. Not. R. Astron. Soc. 397, 763 (2009).

[87] M. Tanabashi et al. (Particle Data Group), Phys. Rev. D 98, 030001 (2018).

[88] M. Srednicki, Nucl. Phys. B260, 689 (1985).

[89] M. Bauer, M. Neubert, and A. Thamm, J. High Energy Phys. 12 (2017) 044.

[90] D. G. Yakovlev and K. P. Levenfish, Astron. Astrophys. 297, 717 (1995), https://ui.adsabs.harvard.edu/abs/1995A \%26A...297..717Y/abstract.

[91] A. Potekhin, D. Zyuzin, D. Yakovlev, M. Beznogov, and Y. Shibanov, Mon. Not. R. Astron. Soc. 496, 5052 (2020).

[92] D. Page, NSCool: Neutron star cooling code, Astrophysics Source Code Library (2016), ascl:1609.009.

[93] A. Akmal, V. R. Pandharipande, and D. G. Ravenhall, Phys. Rev. C 58, 1804 (1998).

[94] A. Y. Potekhin, D. G. Yakovlev, G. Chabrier, and O. Y. Gnedin, Astrophys. J. 594, 404 (2003).

[95] J. F. Perez-Azorin, J. A. Miralles, and J. A. Pons, Astron. Astrophys. 451, 1009 (2006).

[96] V. E. Zavlin, Theory of radiative transfer in neutron star atmospheres and its applications, in Neutron Stars and Pulsars, edited by W. Becker (Springer, Berlin, Heidelberg, 2009), pp. 181-211.
[97] A. Y. Potekhin, W. C. G. Ho, and G. Chabrier, Proc. Sci., MPCS2015 (2016) 016 [arXiv:1605.01281].

[98] F. Haberl, C. Motch, V. E. Zavlin, K. Reinsch, B. T. Gaensicke, M. Cropper, A. D. Schwope, R. Turolla, and S. Zane, Astron. Astrophys. 424, 635 (2004).

[99] B. Posselt, S. B. Popov, F. Haberl, J. Truemper, R. Turolla, and R. Neuhaeuser, Astrophys. Space Sci. 308, 171 (2007).

[100] W. C. G. Ho, D. L. Kaplan, P. Chang, M. van Adelsberg, and A. Y. Potekhin, Mon. Not. R. Astron. Soc. 375, 821 (2007).

[101] N. Sartore, A. Tiengo, S. Mereghetti, A. De Luca, R. Turolla, and F. Haberl, Astron. Astrophys. 541, A66 (2012).

[102] T. Yoneyama, K. Hayashida, H. Nakajima, S. Inoue, and H. Tsunemi, Publ. Astron. Soc. Jpn. 69, 50 (2017).

[103] F. M. Walter, T. Eisenbeiß, J. M. Lattimer, B. Kim, V. Hambaryan, and R. Neuhäuser, Asrophys. J. 724, 669 (2010).

[104] V. Hambaryan, V. Suleimanov, A. D. Schwope, R. Neuhäuser, K. Werner, and A. Y. Potekhin, Astron. Astrophys. 534, A74 (2011).

[105] C. Motch, A. M. Pires, F. Haberl, A. Schwope, and V. E. Zavlin, Astron. Astrophys. 497, 423 (2009).

[106] N. Tetzlaff, T. Eisenbeiss, R. Neuhäuser, and M. M. Hohle, Mon. Not. R. Astron. Soc. 417, 617 (2011).

[107] A. Pires, A. Schwope, F. Haberl, V. Zavlin, C. Motch, and S. Zane, Astron. Astrophys. 623, A73 (2019).

[108] A. D. Schwope, T. Erben, J. Kohnert, G. Lamer, M. Steinmetz, K. Strassmeier, H. Zinnecker, J. Bechtold, E. Diolaiti, A. Fontana, S. Gallozzi, E. Giallongo, R. Ragazzoni, C. de Santis, and V. Testa, Astron. Astrophys. 499, 267 (2009).

[109] A. M. Beloborodov and X. Li, Astrophys. J. 833, 261 (2016).

[110] V. Anastassopoulos et al. (CAST Collaboration), Nat. Phys. 13, 584 (2017).

[111] P. Zyla et al. (Particle Data Group), Prog. Theor. Exp. Phys. 2020, 083C01 (2020).

[112] N. Bar, K. Blum, and G. D'Amico, Phys. Rev. D 101, 123025 (2020).

[113] B. Posselt and G. Pavlov, Astrophys. J. 864, 135 (2018).

[114] V. I. Kondratiev, M. A. McLaughlin, D. R. Lorimer, M. Burgay, A. Possenti, R. Turolla, S. B. Popov, and S. Zane, Astrophys. J. 702, 692 (2009).

[115] A. Treves, R. Turolla, S. Zane, and M. Colpi, Publ. Astron. Soc. Pac. 112, 297 (2000).

[116] D. Barret et al., Proc. SPIE Int. Soc. Opt. Eng. 9905, 99052F (2016).

[117] C. S. Reynolds, M. C. D. Marsh, H. R. Russell, A. C. Fabian, R. Smith, F. Tombesi, and S. Veilleux, Astrophys. J. 890, 59 (2020).

[118] M. C. D. Marsh, H. R. Russell, A. C. Fabian, B.P. McNamara, P. Nulsen, and C. S. Reynolds, J. Cosmol. Astropart. Phys. 12 (2017) 036.

[119] A. Spector (ALPS Collaboration), arXiv:1611.05863.

[120] E. Armengaud et al. (IAXO Collaboration), J. Cosmol. Astropart. Phys. 06 (2019) 047. 